\title{
Aproximación toxicológica a los psicoestimulantes utilizados con fines recreativos
}

\author{
Aurelio luna Maldonado \\ Catedrático de Medicina Legal y Forense. Universidad de Murcia \\ Enviar correspondencia: Aurelio Luna Maldonado. Departamento de Medicina Legal y Forense. Facultad de Medicina. Universidad de
} Murcia.30100 Espinardo. Murcia. E-mail: aurluna@um.es

\begin{abstract}
Resumen
Este amplio grupo de psicoestimulantes comprende diversas sustancias de diferente estructura quími$\mathrm{ca}$, con cinco mecanismos de acción diferentes simpáticomiméticos. Los psicoestimulantes sintéticos más utilizados pertenecen, principalmente, al grupo de las feniletilaminas y de las arexilaminas. El análisis toxicológico de las sustancias tiene interés tanto desde el punto de vista sanitario como policial (identificación de laboratorios clandestinos, rutas y cauces de distribución, etc.). Las sustancias encontradas en las muestras ilícitas pueden clasificarse en seis grupos: principios activos, disolventes o excipientes, adulterantes, contaminantes, impurezas de fabricación e impurezas de origen. La riqueza en principios activos de los preparados clandestinos de MDMA y análogos ha ido en aumento en la última década, pasando de un $27 \%$ en 1990 hasta el $42 \%$ en 2001. El $82 \%$ de las muestras de MDMA y análogos analizadas, en nuestro medio, carecen de adulterantes, siendo los más frecuentes cafeína, anfetaminas y efedrina. La existencia de fenómenos de tolerancia dificulta el establecimiento de niveles tóxicos y/o letales de estas sustancias.
\end{abstract}

Palabras clave: psicoestimulantes, ocio, toxicología, toxicidad, letalidad

\section{Summary}

This large group of psychostimulants includes several substances with different chemical structures, and five different sympathomimetic mechanisms of action. The most widely used synthetic psychostimulants belong predominantly to the phenylethylamine and arexilamine groups. The toxicological analysis of these substances is of interest from both a health and police perspective (identification of clandestine laboratories, distribution routes and pathways, etc.). The substances detected in the illicit samples can be classified into six groups: active ingredients, solvents, or excipients, adulterating substances, contaminating substances, manufacturing impurities, and original impurities. The wealth of active ingredients contained in clandestine MDMA preparations and their analogues have increased in the last decade, from $27 \%$ in 1990 up to $42 \%$ in 2001. Eighty-two percent of the MDMA and analogue samples analysed to date in our area have not been adulterated; of those samples that have been adulterated, mainly caffeine, amphetamines, and ephedrine had been added. Tolerance phenomena make it difficult to establish toxic and/ or lethal levels for these substances.

Key words: psychostimulants, leisure, toxicology, toxicity, lethality

\section{INTRODUCCION}

os psicoestimulantes constituyen un grupo amplio de sustancias de diversa estructura química que tienen como nexo común la capacidad de producir una estimulación de la actividad de diversas estructuras funcionales del sistema nervioso que se expresan por un cuadro de estimulación simpática, sola o asociada a otros efec- 
tos, generada a través de una serie de interacciones en los neurotransmisores y/o en sus receptores. Los agentes simpaticomiméticos producen sus efectos a través de 5 mecanismos diferentes, que pueden coincidir más de uno en algunas sustancias (Kolecki, 2001):

- El primer mecanismo implica la estimulación directa de los receptores alfa y beta adrenérgicos.

- El segundo mecanismo implica la liberación de noradrenalina desde el citoplasma presináptico por un proceso de exocitosis.

- El tercer mecanismo implica la estimulación directa de los receptores adrenérgicos y una liberación indirecta de noradrenalina presináptica.

- Otro mecanismo es el bloqueo de la recaptación presináptica de noradrenalina.

- Una última posibilidad es el bloqueo enzimático de la degradación de la noradrenalina.

Se trata de sustancias que se encuentran en plena fase de expansión en su consumo ya que sus efectos se ajustan a las expectati- vas de los consumidores y responden a los requerimientos de un mercado muy activo. Históricamente es posible establecer ciclos de expansión y disminución en el consumo de estas sustancias (Sánchez-Ramos, 1993). Por otro lado la relativa facilidad en la fabricación de algunos de estos compuestos (anfetaminas, MDMA, etc.) permite la localización de los laboratorios clandestinos en zonas próximas a las de su distribución y consumo y pueden pasar fácilmente inadvertidos debido a que la infraestructura necesaria puede ubicarse y camuflarse sin ninguna dificultad.

Los psicoestimulantes pueden clasificarse en:

A) Sintéticos: Anfetaminas y derivados (MDMA, MDA, etc.), Metilfenidato, Fenilpropanolamina, Fenfluramina

B) Naturales: Cocaína, Absenta, Cafeína, Efedrina, Khat, etc.

Los psicoestimulantes sintéticos mas utilizados se especifican en la Tabla 1 (Roig Traver, 1989; Cabrera Bonet y col, 1994; Leiva del Palacio y col, 1996).

\begin{tabular}{|l|}
\hline \multicolumn{1}{|c|}{ Tabla 1: Principales psicoestimulantes sintéticos } \\
\hline Derivados de las FENILETILAMINAS: \\
\hline Anfetaminas \\
Metanfetamina. \\
TMA-2 (2,4,5-trimetoxianfetamina) \\
DOM, STP (4 metil-2,5-dimetoxianfetamina) \\
PMA (parametoxianfetamina) \\
DOB (4-bromo-2,5-dimetoxianfetamina) \\
MDA (3,4 metilendioxianfetamina) \\
MDMA (3,4 metilendioximetanfetamina) \\
MDE (n-etil metilendioxianfetamina) \\
DOET (2,5-dimetoxi-4-etilanfetamina) \\
DMA (2,5-dimetoxianfetamina) \\
MMDA (3-metoxi-4,5-metilendioxianfetamina) \\
\hline Derivados de las AREXILAMINAS: \\
\hline Fenciclidina \\
PCC (piperidinociclohexanocarbonitrilo) \\
TCP (tiofenofenciclidina) \\
PCE (n-etil fenciclidina) \\
PHP (fenilciclohexilpirrolidina ) \\
\hline Otros \\
\hline Aminorex y 4-metilaminorex \\
Catinona y metcatinona \\
Pemolina \\
Oxibato sódico (gamma hidroxibutirato, GHB) \\
\hline
\end{tabular}




\section{FENILETILAMINAS}

Las Feniletilaminas constituyen en la actualidad el grupo más amplio de sustancias psicodélicas conocidas existiendo tanto derivados naturales como sintéticos (Roig Traver, 1989).

Los derivados sintéticos constituyen un grupo con una gran implantación en su consumo, se conocen de forma genérica como "drogas de diseño"- denominados también anfetaminas alucinógenas o anfetaminas de anillo sustituido. De entre todas las drogas de diseño las de uso más común,con diferencia del resto, son los derivados de la feniletilamina, y entre ellos la 3,4 metilenedioximetanfetamina (MDMA), variante metoxilada de la metanfetamina, conocida como "Éxtasis", "Adán" y la 3,4 metilendioxianfetamina (MDA) conocida como "droga del amor"; otros compuestos más recientes, como la N-etilmetilendioxianfetamina (MDEA), han adquirido cierta reputación aumentando, por lo tanto, su consumo (Cabrera Bonet y col, 1994), sobre todo por jóvenes.

DOM.- La DOM (STP) fue la primera de esta sustancias que apareció en el mercado ilícito. Sintetizada por Shulgin en 1963 hizo su primera aparición en 1967 con el nombre de STP - serenidad, tranquilidad y paz - (Naciones Unidas, 1988). Su estructura es similar a la de la mescalina y a la anfetamina. En 1977 fue incluida en las listas de convenio sobre sustancias psicotrópicas de 1971 (Naciones Unidas, 1988).

DMA.- La DMA no tiene ningún uso terapéutico, pero como producto químico existe una considerable demanda en la industria fotográfica y es posible que se desvíe alguna cantidad hacia usos ilícitos. En 1986 fue incluida en la lista I del convenio sobre sustancias psicotrópicas de 1971 (Naciones Unidas, 1988).

MMDA.- La MMDA fue sintetizada en 1962 como parte de una serie de compuestos con el fin de investigar la actividad combinada de la mescalina y la anfetamina. Químicamente se parece a la miristicina, principal componente de la nuez moscada y del macis. En 1970 fue incluida en las listas del Canadá y de los EEUU de América y en 1986 fue añadida a la lista I del convenio sobre sustancias psicotrópicas de 1971 (Naciones Unidas, 1988).

TMA.- La TMA fue sintetizada en 1947, siendo un homólogo de la mescalina. Apareció en el mercado ilícito en los años setenta. En 1986 fue incluida en la lista I del convenio sobre sustancias psicotrópicas de 1971 (Naciones Unidas, 1988).

MDA.- La MDA fue sintetizada en 1910, y estudiada farmacológicamente en investigaciones con animales en 1939. Ha sido estudiada como droga que suprime el apetito como fármaco antitusígeno y ataráxico y como antidepresivo; sin embargo, nunca llegó a comercializarse (Naciones Unidas, 1988). En los años cuarenta se observaron sus propiedades psicoactivas y sus efectos característicos sobre el sistema nervioso central. Su uso fue declarado ilegal en 1970 y en 1985 fue incluida en la lista I del convenio sobre sustancias psicotrópicas de 1971 (Naciones Unidas, 1988).

MDMA.- El MDMA, 3.4 metilendioximetanfetamina, es una variante metoxilada de la metanfetamina (Cami, 1995). Genéricamente el MDMA puede considerarse un derivado de la feniletilamina igual que la mescalina y la anfetamina. La adicción de un grupo metilo en el - $\mathrm{NH} 2$ junto a la presencia en el anillo bencénico del grupo metilén dioxi, origina el MDMA. Fue desarrollado originalmente en Alemania en 1914, el primer año de la primera guerra mundial. Fue patentado por los laboratorios Merck Pharmaceutical Company como un supresor del apetito, no llegando a comercializarse, y luego quedo en el olvido (Eisner, 1995; Cami, 1995). En 1953-1954 fue una de las sustancias junto con el MDA y otras utilizadas en diferentes pruebas por la $U$ $S$ Navy en Maryland quien lo codificó con el $n^{\circ}$ EA-1473 y, aunque entonces no se efectuó ninguna investigación en humanos, ya se comprobó su toxicidad en diferentes especies animales. Estos resultados fueron publicados en 1973, veinte años más tarde. Hasta 
dicho año no se publicó el primer informe que indicaba la psicoactividad del MDMA en seres vivos aunque se había iniciado ya la difusión de la droga en la sociedad.

El MDMA fue poco conocido hasta 1984, fecha en que empezaron a publicarse los primeros artículos sobre ella acaparando desde la fecha una gran atención por parte de los medios de comunicación. La primera publicación relativa a la aplicación terapéutica de la MDMA fue la de Greer y Tolbert (1986), en la que describieron los resultados de su experiencia en 29 pacientes realizada en 1983. Greer y Tolbert (1990) defendían que la MDMA era una sustancia de propiedades terapéuticas similares a la LSD, aunque más manejable y exenta de los riesgos del alucinógeno. Se resaltaron los efectos inductores de empatía y facilitadores de la comunicación en sujetos con significativos conflictos emocionales. Greer y Tolbert (1990) también defendieron su utilidad para el tratamiento del alcoholismo y otras dependencias y sostenían su bajo potencial de abuso en condiciones terapéuticas.

Como consecuencia de la gran difusión informativa se produce un gran aumento de la demanda sin que la oferta pueda satisfacerla por lo que comienza a adulterarse con otras sustancias: PCP, MDA, LSD, cafeína, efedrina etc. Debido a estas sustancias adulterantes comienzan a producirse frecuentes urgencias médicas relacionadas con el consumo de esta droga. Aunque en aquel momento existían pocos datos sobre los efectos tóxicos y farmacológicos de la MDMA, se conocía su analogía estructural con la MDA, cuya capacidad para producir una degeneración selectiva de los terminales nerviosos serotoninérgicos en ratas había sido demostrado durante ese mismo año (Ricaurte y col, 1985), lo que provoca que el 1 de Julio de 1985 la DEA incluya, por un procedimiento urgente, al MDMA en la lista I de la Ley de sustancias controladas junto a productos como la Heroína, LSD, MDA y otras sustancias con alto potencial de abuso, ausencia de uso médico conocido e incumplimiento de las normas de seguridad exigidas por la Food and Drug Administration (FDA).
En 1986, la comisión de estupefacientes de las Naciones Unidas, en sesión celebrada en el mes de Febrero, acordó incluir el MDMA, junto con otros productos, en la Lista I del convenio de sustancias psicotrópicas de 1971, lo que quiere decir que está prohibido internacionalmente su fabricación y venta, su uso, importación, tránsito, comercio y tenencia de dichas sustancias, así como de los preparados que la contengan. En España por Orden Ministerial de 30 de Mayo de 1986 se incluyó el MDMA en la lista I, del Anexo I, del Real Decreto 2829/1977, de 6 de Octubre.

\section{ANÁLISISTOXICOLÓGICO}

Vamos a centrarnos por razones obvias de espacio en las sustancias psicoestimulantes que en el momento actual tienen una mayor presencia en el mercado ilícito de nuestro país, nos referimos a la cocaína a las anfetaminas y a las denominadas drogas de diseño (MDA,MDMA, etc.), además de su uso recreativo y dentro de patrones adictivos conviene recordar el consumo de estas sustancias, fundamentalmente, anfetaminas, cocaína y cafeína, en el ámbito concreto de la práctica deportiva con el incremento objetivo de riesgo que esto supone (George, 2000).

El producto final que llega al consumidor, es el resultado de un complejo proceso, que surge con las expectativas de un mercado que demanda unos efectos, y que desde el productor de las materias primas, los que las elaboran, hasta el vendedor que en un esquina vende la "papelina" o el "tripi" o la pastilla de turno, van a dejar su impronta de forma voluntaria en unos casos (adulterantes, excipientes), e involuntaria en otros (contaminantes, e impurezas de síntesis). Hace ya bastantes años, a principios de la década de los 70 , se subrayó la presencia de en las preparaciones ilícitas de adulterantes y excipientes que podían plantear problemas específicos de salud (Johnson y Gunn, 1972; Perry, 1975). 
Las sustancias encontradas en las muestras ilícitas se pueden clasificar en seis grupos:
a) Principios activos
b) Disolventes o excipientes
c) Adulterantes.
d) Contaminantes
e) Impurezas de fabricación
f) Impurezas de origen.

El interés del conocimiento de estos componentes es múltiple y puede estructurarse en los siguientes campos:

a) Sanitario. Conocimiento de los riesgos concretos de las sustancias presentes en el mercado (Luna y col, 1984; Luna, 1997; Smith, 2002).

b) Conocimiento de las expectativas del mercado ilícito (Renfroe y Messinger, 1985; Klatt y col, 1986; Kaa, 1992).

c) Identificación de los laboratorios clandestinos.

d) Conocimiento de las rutas y cauces de distribución de estos productos (Hernández-Cueto y col, 1985; Bermejo-Barrera y col, 1999; Luna, 2001; Chiarotti y col, 2002).

Los contaminantes, (bióticos y abióticos) van a estar presentes de forma accidental (no intencionada) en las muestras, fruto de deficiencias en la manufactura y/o manipulación y en los procesos de transporte y distribución. Su forma de acceso al producto final permite su utilidad en el proceso de identificación de las muestras, ya que al ser un proceso accidental y no controlado por los que intervienen a lo largo de todo el proceso, va a escapar a cualquier forma de control y/o manipulación intencionada para enmascararlo.

Entre los contaminantes bióticos, los más generalizados son diferentes especies de hongos que estudiamos en muestras de heroína y cocaína (Luna y col, 1986; Domínguez y col, 1991), y entre los contaminantes abióticos hemos estudiado la presencia de metales ( $\mathrm{Pb}, \mathrm{Cd}, \mathrm{Cu}, \mathrm{Mn}, \mathrm{Ba}$, etc.) en muestras de heroína. Diversos autores (Infante y col, 1999; Bermejo-Barrera y col, 1999), han estudiado las concentraciones de $\mathrm{Ag}, \mathrm{Al}, \mathrm{Ca}, \mathrm{Cd}$,
$\mathrm{Cr}, \mathrm{Cu}, \mathrm{Fe}, \mathrm{K}, \mathrm{Mg}, \mathrm{Mn}, \mathrm{Na}, \mathrm{Ni}, \mathrm{Pb}, \mathrm{Sr}$ y $\mathrm{Zn}$ en 46 muestras de cocaína, decomisadas en Galicia pudiendo diferenciar su procedencia (Colombia y Venezuela) a través de los perfiles de los elementos estudiados.

Los adulterantes son sustancias que se incorporan de forma intencionada a las muestras, buscando potenciar ciertos efectos o suplir la escasa cantidad de principio activo con una sustancia que pudiera simular sus efectos. El interés del conocimiento de estos compuestos procede de su toxicidad potencial al tener un efecto sinérgico con los principios activos en algunos casos (Renfroe y Messinger, 1985; Shannon, 1988; Quandt y col, 1988; Schauben, 1990) o en otros de su acción tóxica específica, como ejemplos tenemos la producción de metahemoglobina por el consumo de cocaína adulterada con benzocaina (McKinney y col, 1992), las alteraciones renales en las mezclas de heroínacocaína (speed-ball) (Cunningham y col, 1984), o las acciones tóxicas de la fenitoina en muestras de crack adulteradas por este compuesto (Katz y col, 1993).

Por otro lado, muchos adulterantes pueden modificar y/o enmascarar el resultado de los análisis con las técnicas habituales de las muestras de orina de los consumidores de estas sustancias (Mikkelsen y Ash, 1988; Wu y col, 1995).

Las impurezas de síntesis y/o los restos de los productos utilizados en su manufactura, como los residuos de solventes sirven para la identificación de los alijos (Chiarotti y col, 2002), y para la caracterización de los laboratorios clandestinos (Luna, 2001).

Comenzaré con la estudio de la evolución de la composición de las muestras clandestinas de cocaína. En el momento actual es la sustancia psicoestimulante mas utilizada en nuestro medio con exclusión de la cafeína.

La elaboración de la cocaína se realiza a partir de las hojas de eritroxilon coca, que sufre una serie de procesos de transformación, desde la pasta de coca hasta el clorhidrato de benzoilecgonina, donde intervienen, gasolina, solventes orgánicos (éter, acetona, 
etc.), ácido clorhídrico, etc. en donde pueden quedar residuos de las sustancias utilizadas, desde impurezas de síntesis (metil-ecgonina,etc), otros de alcaloides, metales, hasta residuos de solventes.

Los adulterantes se incorporan intencionalmente a la cocaína, con una finalidad de aumentar el rendimiento económico añadiendo sustancia de menor coste. Entre las sustancias empleadas con estos fines tenemos: anestésicos locales (procaína, lidocaína, tetracaína), otros estimulantes (anfetaminas, cafeina, metilfenidato, estricnina), ácido lisérgico y su dietilamida (LSD), fenciclidina (PCP), heroína, y fenitoina (Burnett y Adler, 2002).

Con relación a las muestras de cocaína analizadas por nosotros conviene reseñar la ausencia relativa de impurezas de síntesis en las muestras más recientes, que corresponde a una sistemática analítica más sencilla y eficiente y una mayor capacitación de los manipuladores de la pasta de coca (cocineros). En las muestras del periodo 1991-1994 hemos identificado como impurezas de síntesis metilecgonina $(6,25 \%)$, benzoilecgonina $(4,68 \%)$ y cis-cinnamoilcocaína y trans-cinnamoilcocaína en un $12,5 \%$ de las muestras analizadas, siendo coincidentes con otros autores (Lurie y col, 1987; Le Belle y col, 1987, 1988, 1991; Moore y Casale, 1994). Los porcentajes relativos de cis-cinnamoilcocaína y trans-cinnamoilcocaína, sirven para la identificación de los alijos, diferencias superiores a un 3\% indican un origen distinto (Le Belle y col, 1990, 1991; Janzen y col, 1992).

Con relación a los adulterantes, la evolución de las muestras de cocaína analizadas ofrece una disminución, a lo largo del período estudiado, en el número de sustancias farmacológicamente activas y en la frecuencia de aparición de las mismas, así en el año 1991 el $50 \%$ de las muestras presentaban alguna sustancia adulterante (Anfetamina 16,6\%, Lidocaína 16,6\%, Cafeína 16,6\% y Paracetamol 16,6\%) en el año 1994 solo un $11 \%$ de todas la muestras presentaban algún adulterante (López-Ros y col, 1997) y en el año 2001, el 100\% estaban libres de adulterantes (Luna, 2001). Las preparaciones actuales en el mercado ilícito de cocaína en EEUU, presentan adulterantes en un bajo porcentaje, así del 8-20\% de los estimulantes presentes en la calle contienen cocaína y clorhidrato de metanfetamina (Burnett y Adler, 2002). Lo que demuestra una mayor facilidad en la llegada de esta sustancia con unos niveles de oferta bastante amplios, que no hacen necesarios los procesos de adulteración.

Las muestras de heroína + cocaína (speedballs), analizadas en nuestro medio, contienen una escasa presencia de adulterantes farmacológicamente activos, siendo los más frecuentes la cafeína y la procaína.

La evolución cronológica demuestra una disminución evidente en el número y frecuencia de asociaciones; la explicación vendría dada, como hemos manifestado anteriormente, por las condiciones del mercado, con un incremento en la calidad y pureza de las muestras ilícitas, tanto de las de heroína como de las de cocaína.

En las muestras de cocaína en EEUU, Insley y col (1986), refieren como los adulterantes más comúnmente usados los anestésicos locales (lidocaína, benzocaina, etc.), y otras sustancias como anfetaminas, fenciclidina y estricnina. En un estudio realizado en Los Angeles (período 1971-1980), los adulterantes más frecuentes encontrados fueron cafeína, efedrina, lidocaína y procaína (Klatt y col, 1986). En otros estudios en este periodo se describen la presencia de cafeína, efedrina, fentermina, fenil-propanol-amina, lidocaína, benzocaína, procaína, tetracaína, aspirina, etc. (Shannon, 1988).

En nuestro país en el periodo entre septiembre de 1985 a Mayo de 1987, Gómez y Rodríguez (1989), encuentran que el 59,6\% de las muestras de cocaína analizadas presentaban algún adulterante, siendo el adulterante más frecuente (el 52\%) la lidocaína. En el conjunto de las muestras de cocaína son escasas las asociaciones de principios activos, lo habitual es que, en el caso de que esté adulterada, aparezca una sola sustancia farmacológicamente activa distinta de la cocaína. Fucci y De Giovanni (1998), en un estudio realizado en Roma, en 156 muestras 
ilícitas de cocaína entre Junio de 1996 y Junio de 1997, encontraron como los adulterantes más frecuentes la lidocaína $(14,7 \%)$ y la cafeína (9\%). En nuestro medio nuestros resultados son concordantes con los anteriores, en el momento actual la pureza de las muestras de cocaína es alto siendo muy escasas las muestras que presentan adulterantes.

Clásicamente, la elección de un excipiente se realiza en base a dos cualidades esenciales: la primera, que sea de color semejante al principio activo, para dar sensación de mayor cantidad de sustancia al consumidor, y que no distorsione o atenúe los efectos del principio activo y pueda administrarse sin problemas por la vía de administración habitual; la segunda, es su fácil disponibilidad y su precio asequible.

La vía de administración condiciona el tipo de excipiente que puede emplearse, así muchas de las sustancias que por vía oral no plantean ningún problema están absolutamente contraindicadas en la vía endovenosa por los efectos adversos que pueden producir (tromboflebitis, embolias, etc.), determinados azucares no son el excipiente o diluyente para sustancias que vayan a consumirse fumadas por los caracteres organolépticos que proporcionan al humo o por los residuos de la combustión que limitan la difusión de los principios activos.

Los excipientes mas utilizados han sido los azucares, dextrosa, sucrosa, lactosa, manitol (Shannon, 1988). Otros excipientes utilizados han sido el silicato magnésico, el ácido ascórbico, el ácido bórico, etc. (Burnett y Adler, 2002).

En el primer año analizado por nosotros, 1991, destacan la lactosa y el manitol con un porcentaje muy elevado frente al resto. En los años posteriores, 1992,1993 y siguientes el manitol desplaza a la lactosa (López-Ros y col, 1997). Esta tendencia se ha venido manteniendo hasta la actualidad, destaca el gran aumento del manitol que pasa de un $9 \%$ en 1988, casi un 60 \% en el año 1993 y más del 70\% en el año 2001 (Luna, 2001).
En las asociaciones de excipientes en muestras de cocaína, se observa un bajo número de éstas comparado con lo encontrado en las muestras de heroína, predominando un sólo excipiente, lo que está en concordancia con los resultados que evidencian una menor adulteración de la cocaína (López-Ros y col, 1997).

Una huella analítica completa debe incluir dentro de los contaminantes los metales y los hongos, ya que unidos presentan una gran capacidad de resolución en la caracterización de las muestras y por otra parte las impurezas de síntesis, los principios activos, los adulterantes y los excipientes, lo que proporciona una auténtica huella química que permite identificar y caracterizar una muestra y conocer con detalle los cauces de comercialización y distribución.

La síntesis de metanfetamina es relativamente sencilla pudiendo realizarse en laboratorios clandestinos que pueden montarse en cualquier lugar (cocinas, remolques, roulots, etc.), es un derivado de la feniletilamina, la vía de síntesis habitualmente utilizada es a partir de la L-efedrina con fosforo rojo en medio ácido. A veces puede sustituirse la efedrina por clor-efedrina o por metil-efedrina. Las impurezas detectadas han sido, estereoisómeros como el N-metil-N-(alfa- metilfeniletil)amino-1-fenil-2-propanona y el derivado ciscinnamoil de la metanfetamina (Windahl y col, 1995). En nuestros análisis hemos detectado las siguientes impuerezas de síntesis en las diferentes drogas de diseño, ácido hexadecanóico (palmítico), oleamida, pirimidina, isopropilamina, 3-4 metilen-dioxi-fenil-cetona (Sánchez-Sennac y col, 2002).

Las muestras ilícitas de metanfetaminas pueden estar contaminadas por compuestos orgánicos e inorgánicos, incluso por metales pesados como el plomo o el mercurio (Derlet y Albertson, 2002).

Los laboratorios clandestinos de anfetaminas y derivados suelen situarse en las zonas próximas a las del consumo. En EEUU, el $75 \%$ de los laboratorios detectados han sido en las grandes ciudades de California, Tejas, y Oregon. A partir de Agosto de 1999, los labo- 
ratorios se desplazaron a la zona Mejicana de la frontera con EEUU, el precio en esta zona de las anfetaminas era de 26.000 dólares por kilo (el precio de la cocaina es de alrededor de14.000 dólares/kg) (Handly, 2002). En los últimos años asistimos a un incremento de su consumo en EEUU (Albertson y col, 1999). En nuestro país los decomisos de anfetaminas y meta-anfetaminas son proporcionalmente menores a los de MDMA y MDA.

Las modificaciones de la presencia de estas sustancias muestran unas amplias fluctuaciones derivadas de los cambios en las expectativas y en los patrones del consumo. Así, la presencia de las anfetaminas en el mercado danés fue muy escasa en el periodo 1982-1984, sin embargo, subió vertiginosamente casi al 50\% de las muestras ilícitas de drogas de abuso analizadas en el año 1987 (Kaa, 1988, 1992). En nuestro país un estudio sobre las muestras de sustancias analizadas en el Instituto Nacional de Toxicología en Madrid en el periodo de Septiembre de 1985 a Mayo de 1987, de un total de 414 muestras procedentes del Mercado ilícito la distribución fue del $63.5 \%$ contenían heroína, el $12.5 \%$ cocaína, el $8.5 \%$ anfetaminas y el $15.4 \%$ otras sustancias, (Gómez y Rodríguez, 1989). Por otra parte, en este periodo la presencia de adulterantes era prácticamente la norma, así por ejemplo, en las muestras antes descritas se detectaron adulterantes en el $56 \%$ de las muestras de anfetaminas (Gómez y Rodríguez, 1989).

El consumo de las drogas de diseño ha iniciado una fase de consolidación del mercado clandestino después de un periodo de rápida expansión a partir de la primera mitad de la década de los noventa, el sudeste español es una zona importante de producción y consumo de estas sustancias, por lo que resulta muy interesante el estudio de los productos presentes en el mercado ilícito, como expresión de las características del mercado clandestino (expectativas de los consumidores y ofertas de los productores).

En un trabajo anterior (Luna y SánchezSennac, 1997), iniciamos una aproximación a este problema, encontrándonos con un mer- cado clandestino dinámico en continua evolución con una diversificación de los productos presentes en el mismo y con una mayor pureza en las preparaciones y una mayor riqueza en los principios activos. En un reciente trabajo (Sánchez-Sennac y cols, 2002), hemos podido confirmar las tendencias anteriormente descritas

Desde una perspectiva práctica vamos a centrarnos en el MDMA, la sustancia más frecuentemente consumida en nuestro país y que representa en torno al $50 \%$ de las sustancias presentes en el mercado ilícito de estos compuestos (Luna y Sánchez Sennac, 1997; Sánchez Senac y col, 2002). El número de los alijos de las distintas sustancias estudiadas en nuestro medio, se observa en la tabla 2.

\begin{tabular}{|lll|}
\hline \multicolumn{3}{|c|}{$\begin{array}{c}\text { Tabla 2: Alijos de las diferentes sustancias } \\
\text { estudiadas en nuestro medio }\end{array}$} \\
Sustancia & Número de alijos & $\%$ \\
\hline Piracetan & 1 & 1,04 \\
\hline Codeina & 1 & 1,04 \\
\hline Metanfetamina & 1 & 1,04 \\
\hline Efedrina & 2 & 2,08 \\
\hline Mescalina & 1 & 1,04 \\
\hline Cafeína & 2 & 2,08 \\
\hline MDMA & 38 & 39,58 \\
\hline MDA & 11 & 11,45 \\
\hline MDE & 26 & 27,08 \\
\hline MDEA & 2 & 2,08 \\
\hline MDMA/MDA & 9 & 9,37 \\
\hline 2-CB & 2 & 2,08 \\
\hline
\end{tabular}

Nuestros resultados son coincidentes con los obtenidos en Irlanda por O'Connell y Heffron (2000), aún cuando estos autores detectan la presencia de N-metil-1-feniletilamina, sustancia no detectada en nuestras muestras. La riqueza en principios activos de los preparados clandestinos en nuestro medio ha ido en aumento desde una media del $27 \%$ en los años 1990-91 hasta el 42\% en el año 2001 (Sánchez-Sennac y col, 2002). El 82\% de las muestras analizadas en nuestro medio, carecen de adulterantes. En la Tabla 3 se resumen los adulterantes más frecuentemente encontrados. 


\begin{tabular}{|c|c|c|c|c|c|}
\hline \multicolumn{6}{|c|}{$\begin{array}{l}\text { Tabla 3: Adulterantes más } \\
\text { frecuentemente detectados }\end{array}$} \\
\hline MDMA & & MDA & & MDEA & \\
\hline Sin adulterantes & $63 \%$ & Sin adulterantes & $75 \%$ & Sin adulterantes & 83,33 \\
\hline Cafeína & $15 \%$ & Cafeína & $17 \%$ & Cafeína & 10 \\
\hline D-Anfetamina & $8 \%$ & Imidazol & $8 \%$ & Efedrina & 6,66 \\
\hline Efedrina & $6 \%$ & & & & \\
\hline Acido Acetilsalicílico & $4 \%$ & & & & \\
\hline Piracetan & $2 \%$ & & & & \\
\hline
\end{tabular}

\begin{tabular}{|c|c|c|c|c|c|}
\hline \multicolumn{6}{|c|}{ Tabla 4: Excipientes encontrados en las distintas muestras } \\
\hline MDMA & & MDA & & MDEA & \\
\hline Lactosa & $67,5 \%$ & Lactosa & $66,6 \%$ & Lactosa & $60 \%$ \\
\hline Manitol & $5,4 \%$ & Lactosa/sepiolita & $11,1 \%$ & Lactosa/Sepiolita & $4 \%$ \\
\hline Lactosa/Yeso & $2,7 \%$ & & & Glucitol & $4 \%$ \\
\hline Arabinosa/Sorbitol & $5,4 \%$ & & & Lactosa/Sacarina & $4 \%$ \\
\hline Lactosa/Sorbitol & $10,8 \%$ & & & Lactosa/Sorbitol & $8 \%$ \\
\hline Lactosa/Talco & $2,7 \%$ & Lactosa/Talco & $11,1 \%$ & Lactosa/Talco & $12 \%$ \\
\hline Lactosa/Manitol & $5,4 \%$ & Lactosa/Manitol & $11,1 \%$ & Sorbitol & $4 \%$ \\
\hline Manitol/Talco & $2,7 \%$ & & & Manitol/Talco & $4 \%$ \\
\hline
\end{tabular}

El excipiente mas empleado en el mercado ilícito es la lactosa que está presente en más del $70 \%$ de las muestras (Sánchez-Sennac y col, 2002) (Tabla 4).

Las características del mercado clandestino y el acceso a los distintos productos que actúan como precursores en su síntesis y a los excipientes, van a condicionar muchas de las características finales del producto.

Un problema de difícil solución es la interpretación de los resultados analíticos. La gran sensibilidad de los métodos analíticos existentes en la actualidad permiten la detección de concentraciones muy bajas de estas sustancias y/o sus metabolitos en los diferentes medios biológicos, hasta 5-30 partes por billón para la cocaína (Traldi y col, 1993). En el momento actual resulta posible el control del consumo de estas sustancias (cafeína, cocaina, anfetaminas y derivados, etc.) a través del estudio de las concentraciones de estas sustancias en el pelo (Luna, 1991; Traldi y col, 1993; Tagliaro y col, 1993; Miller y col, 1997; Rothe y col, 1997).
Los fenómenos de tolerancia determinan una gran oscilación entre los valores que podemos definir como tóxicos, en la medida que determinan un cuadro de intoxicación aguda. Por otra parte, no existen unos límites establecidos para la Dosis Letal 50. La Dosis Letal 50 del MDMA ha sido calculada para las ratas en $49 \mathrm{mg} / \mathrm{Kg}$, para los perros en 14 $\mathrm{mg} / \mathrm{Kg}$ y de $22 \mathrm{mg} / \mathrm{Kg}$ en los macacos rhesus, no existiendo una DL50 calculada para el hombre. Diversos autores (Yev y In-Hei, 2002; Raikos y col, 2002) encuentran valores en sangre de $2 \mu \mathrm{g} / \mathrm{ml}$ en intoxicaciones mortales por MDMA. Se trata, en cualquier caso, de un diagnóstico que requiere interpretar los resultados analíticos en el contexto de los hallazgos de autopsia. Al tratarse de sustancias que se comportan como bases débiles van a sufrir procesos de redistribución postmortem que obligan a tener presente el origen de la sangre para una adecuada interpretación de los resultados. Vanbinst y col (2002), encuentran oscilaciones en las concentraciones de estas sustancias, según el medio biológico utilizado (Tabla 5). 
Tabla 5: Concentración de distintos psicoestimulantes según el medio biológico utilizado

\begin{tabular}{|c|c|c|c|c|c|c|c|c|}
\hline Sustancia & N & $\begin{array}{l}\text { Sangre del } \\
\text { S.N.C } \mu \mathrm{g} / \mathrm{ml}\end{array}$ & N & $\begin{array}{l}\text { Sangre cardíaca } \\
\mu \mathrm{g} / \mathrm{ml}\end{array}$ & N & $\begin{array}{l}\text { Sangre de la } \\
\text { vena femoral } \\
\mu \mathrm{g} / \mathrm{ml}\end{array}$ & N & $\begin{array}{l}\text { Bilis } \\
\mu \mathrm{g} / \mathrm{ml}\end{array}$ \\
\hline Anfetaminas & 1 & 1,153 & 1 & 0,693 & 1 & 0,593 & 1 & 1,373 \\
\hline MDMA & 2 & $0,329-0,865$ & 1 & 0,279 & 2 & $0,29-0,404$ & 3 & $\begin{array}{l}0,222-1,279 \\
(0,707)\end{array}$ \\
\hline Cocaína & 9 & $\begin{array}{l}0,014-6,363 \\
(1,702)\end{array}$ & 3 & $\begin{array}{l}0,012-5,143 \\
(1,919)\end{array}$ & 4 & $\begin{array}{l}0,31-7,775 \\
(2,836)\end{array}$ & 9 & $\begin{array}{l}0,29-34,06 \\
(7,89)\end{array}$ \\
\hline Benzoilecgonina & 11 & $\begin{array}{l}0,036-7,337 \\
(1,636)\end{array}$ & 5 & $\begin{array}{l}0,077-9,27 \\
(2,774)\end{array}$ & 4 & $\begin{array}{l}1,94-10,98 \\
(4,317)\end{array}$ & 12 & $\begin{array}{l}0,252-67,30 \\
(9,99)\end{array}$ \\
\hline
\end{tabular}

\begin{tabular}{|lllll|}
\hline & $\begin{array}{c}\text { Tabla 6: Concentraciones en sangre de drogas de síntesis (pacientes fallecidos) } \\
\text { Sangre femoral } \mu \mathrm{g} / \mathrm{ml}\end{array}$ & Sangre del corazón derecho $\mu \mathrm{g} / \mathrm{ml}$ \\
\hline & MDMA & MDA & MDMA & MDA \\
\hline Caso1 & 2750 & 280 & 9100 & 830 \\
\hline Caso 2 & 1564 & 43 & 417 & 37 \\
\hline
\end{tabular}

Karch (2002), encuentra las siguientes concentraciones en dos casos de fallecidos con presencia de MDMA y MDA en su sangre (Tabla 6).

Como podemos observar existe una mayor concentración en la sangre del corazón derecho, provocada por un artefacto derivado de la redistribución post-mortem de estas sustancias y su liberación de los tejidos a la sangre al producirse la acidosis propia de los procesos de autolisis.

En el caso de la cocaína nos encontramos en una situación similar. Al comienzo de los años 1980 se admitía como una dosis letal, concentraciones en sangre de $5 \mathrm{mg} / \mathrm{l}$, posteriormente se han publicado casos de sujetos fallecidos por traumatismos con cifras superiores a los 5mg/l. Karch (2002), cita un caso de un varón asesinado por un disparo, mientras bebía en un bar, sin que presentara trastornos objetivables, que tras la autopsia y los análisis correspondientes tenía una concentración de cocaína en sangre de 30mg/l.

En la Tabla 7 se reproducen los valores publicados por Winek y col (2001) en una amplia serie de casos.

\begin{tabular}{|lllllll|}
\hline \multicolumn{7}{|c|}{ Tabla 7: Significación de los niveles de una serie de sustancias utilizadas } \\
con fines no terapéuticos (recreativos y/o adictivos) \\
Niveles en sangre
\end{tabular}




\begin{tabular}{|lllllll|}
\hline \multicolumn{7}{|c|}{ Niveles en sangre } \\
\hline Sustancia & \multicolumn{7}{l|}{ Terapéuticos o normales } & $\begin{array}{l}\text { Tóxicos } \\
\mathrm{mgr} \%\end{array}$ & $\mu \mathrm{g} / \mathrm{ml}$ & $\mu \mathrm{g} / \mathrm{ml}$ & $\begin{array}{l}\text { Letales } \\
\mathrm{mgr} \%\end{array}$ & $\mu \mathrm{g} / \mathrm{ml}$ \\
\hline & $0,00003-0,0019$ & $0,0003-0,019$ & - & - & 0,189 & 1,89 \\
\hline Escopolamina & $0,02-0,63$ & $0,2-6,3$ & - & - & - & - \\
\hline Ketamina & - & - & - & - & $0,18-2,6$ & $1,8-26$ \\
\hline MDA & $0,001-0,005$ & $0,01-0,05$ & $0,06-0,5$ & $0,6-5,0$ & $>1$ & $>10$ \\
\hline Metanfetamina & $0,007-0,080$ & $0,07-0,80$ & 0,5 & 5 & $0,8-2,0$ & $8-20$ \\
\hline Meperidina & - & - & - & - & $0,0002-0,0011$ & $0,002-0,011$ \\
\hline Metilfentanil & - & - & - & - & $0,02-0,49$ & $0,2-4,9$ \\
\hline PMA & - & - & $0,0007-$ & $0,007-0,24$ & $0,1-0,5$ & $1-5$ \\
\hline PCP & 0,001 & 0,01 & - & - & $0,2-0,3$ & $2-3$ \\
\hline Propilhexedrina & $0,023-0,107$ & $0,23-1,07$ & $0,03-0,06$ & $0,3-0,6$ & $0,1-1,7$ & $1-17$ \\
\hline Propoxifeno & $0,05-0,077$ & $0,5-0,77$ & - & - & 1,9 & 19 \\
\hline Pseudoefedrina & & & & & & \\
\hline
\end{tabular}

\section{REFERENCIAS}

1. Albertson TE, Derlet RW, Van Hoozen BE. Methamphetamine and the expanding complications of amphetamines. West J Med 1999;170: 214-219.

2. Bermejo-Barrera P, Moreda-Pineiro A, MoredaPineiro J, Bermejo-Barrera A, Bermejo-Barrera AM. A study of illicit cocaine seizure classification by pattern recognition techniques applied to metal data. J Forensic Sci 1999; 44: 270274.

3. Burnett LB, Adler J. Toxicity, cocaine. Emedicine, July 26, 2002

4. Camí J. Farmacologia y toxicidad de la MDMA (Extasis). Barcelona: Ediciones en Neurociencias, 1995.

5. Chiarotti M, Marsili R, Moreda-Pineiro A. Gas chromatographic-mass spectrometric analysis of residual solvent trapped into illicit cocaine exhibits using head-space solid-phase microextraction. J Chromatogr B Analyt Technol Biomed Life Sci 2002; 772: 249-256.

6. Cunningham EE, Venuto RC, Zielezny MA. Adulterants in heroin/cocaine: implications concerning heroin-associated nephropathy. Drug Alcohol Depend 1984; 14: 19-22.

7. Denborough MA, Hopkinson KC. Dantrolene and "ecstasy". Med J Aust 1997; 166: 165-166.

8. Derlet R, Albertson T. Toxicity, Methamphetamine. Emedicine, January 22, 2002.
9. Dominguez E, Infante F, Luna A. Micocontamination of illicit samples of heroin and cocaine as indicator of adulteration. J Forensic Sci 1991; 36: 844-856.

10. Eisner B. Extasis: historia del MDMA. BarceIona: Ediciones Obelisco, 1995.

11. Fucci N, De Giovanni N. Adulterants encountered in the illicit cocaine market. Forensic Sci Int 1998; 95: 247-252.

12. George AJ. Central nervous system stimulants. Baillieres Best Pract Res Clin Endocrinol Metab 2000; 14: 79-88.

13. Gómez J, Rodriguez A. An evaluation of the results of a drug sample analysis. Bull Narc 1989; 41: 121-126.

14. Greer G, Tolbert R. Subjective reports of the effects of MDMA in a clinical setting. $\mathbf{J}$ Psychoactive Drugs 1986; 18: 319-327.

15. Greer G, Tolbert R. The therapeutic use of MDMA. In: Peroutka SJ, ed. Ectasy: The clinical pharmacological and neurotoxicological effects of the drug MDMA. Boston: Kluwer Academic Pub, 1990. pp 21-35.

16. Handly N. Toxicity, Amphetamine. Emedicine, January 21, 2002.

17. Hernández-Cueto C, Luna A, Villanueva E. Niveles de plomo en muestras de heroína. ¿Método para la identificacion de diferentes muestras?. Revista de Toxicología 1985; 2: 115-120.

18. Infante F, Domínguez E, Trujillo D, Luna A. Metal contamination, in illicit samples of heroin. J Forensic Sci 1999; 44: 110-113. 
19. Insley BM, Grufferman S, Ayliffe HE. Thallium poisoning in cocaine abusers. Am J Emerg Med 1986; 4: 545-548.

20. Janzen KE, Walter L, Fernando AR. Comparison análisis of i illicit cocaíne samples. J Forensic Sci 1992; 37: 436-445.

21. Johnson DW, Gunn JW Jr. Dangerous drugs: adulterants, diluents, and deception in street samples. J Forensic Sci 1972; 17: 629-639.

22. Kaa E. Drug abuse in Denmark (Jutland and Funen). A forensic study based upon drugs seized in 1982-1987. Z Rechtsmed 1988; 101 : 229-236.

23. Kaa E. Drug abuse in western Denmark during the eighties. I. Drugs of abuse. Forensic Sci Int 1992; 55: 67-74.

24. Karch SB. Karch's Pathology of Drug Abuse

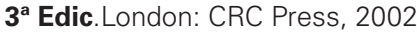

25. Katz AA, Hoffman RS, Silverman RA. Phenytoin toxicity from smoking crack cocaine adulterated with phenytoin. Ann Emerg Med 1993; 22 : 1485-1487.

26. Klatt EC, Montgomery S, Namiki T, Noguchi TT. Misrepresentation of stimulant street drugs: a decade of experience in an analysis program. J Toxicol Clin Toxicol 1986; 24: 441-450.

27. Kolecki P. Toxicity, Sympathomimetic. Emedicine, June 11, 2001.

28. Le Belle M, Lauriault G, Callahan S, Latham D, Chiaarelli $\mathrm{C}$, Beckstead $\mathrm{H}$. The examination of illicit cocaine. J Forensic Sci 1988; 33: 662675.

29. Le Belle MJ, Callahan SA, Latham DJ, Lauriault $\mathrm{G}$. Identification and determination of norcocaine in illicit cocaine and coca leaves by gas-chromatography-MS y HPLC. Analyst 1988; 113: 1213-1215.

30. Le Belle M, Callahanm S, Latham D, Lauriault G, Savard C. Comparasion of illicit cocaine by determination of minor components. J Forensic Sci 1991; 36: 1102-1120.

31. López-Ros I, Perez-Sirvent C, Osuna E, PérezCarceles MD, Luna A. Study of adulterants and diluents presents in Heroin and Cocain street samples. Comunicación presentada al XVII Congreso de la Academia Internacional de Medicina Legal y Social. Dublin, 1997.

32. Luna A, Pla A, Martos M, Villanueva E. Estudio de adulterantes en muestras ilícitas de heroína. Revista de Toxicologia 1984; 1: 125-134.
33. Luna A, Infante F, Domínguez E. Micoflora of potential sanitary interest present in illicit heroin. Z Reschtsmedizin 1986; 96: 297-302.

34. Luna A. Posibilidades periciales de la determinación de sustancias psicoactivas en el pelo. Centro de Estudios Judiciales, 1991; 7: 10-23. Madrid: Ministerio de Justicia.

35. Luna A. La huella analítica en las Drogas de Abuso. Anales de la Real Academia, 1997, pp1-27.

36. Luna A, Sánchez Senac C. Análisis tóxicológico de los comprimidos de MDMA en España. En: Bobes García J, Lorenzo Fernández P, Sáiz Martínez P, eds. Éxtasis (MDMA). Un abordaje comprehensivo. Barcelona: Masson, 1997, pp 73-88.

37. Luna A. La huella analítica en las drogas de abuso. Posibilidades en la identificación de los alijos. Revista de Toxicología 2001; 18: 142.

38. Lurie IS, Moore JM, Cooper DA, Kram TC. Analysis of manufacturing by products and impurities in illicit cocaine via high-performance liquid chromatography and photodiode array detection. J Chromatography 1987; 405: 273281.

39. McKinney CD, Postiglione KF, Herold DA. Benzocaine-adultered street cocaine in association with methemoglobinemia. Clin Chem 1992; 38: 596-597.

40. Mikkelsen SL, Ash KO. Adulterants causing false negatives in illicit drug testing. Clin Chem 1988; 34: 2333-2336.

41. Miller ML, Donnelly B, Martz RM. The forensic application of testing hair for drugs of abuse. NIDA Res Monogr 1997; 167:146-160.

42. Moore JM, Casale JF. In-depth chromatography analyses of illicit cocaine and its precursor, coca leaves. J Chromatography 1994; 674: 165-205.

43. O'Connell D, Heffron JJ. Rapid analysis of illicit drugs by mass spectrometry: results from seizures in Ireland. Analyst 2000; 125: 119-121.

44. Palhol F, Boyer S, Naulet N, Chabrillat M. Impurity profiling of seized MDMA tablets by capillary gas chromatography. Anal Bioanal Chem 2002; 374: 274-281.

45. Perry DC. Editorial. Heroin and cocaine adulteration. Clin Toxicol 1975; 8: 239-243.

46. Quandt CM, Sommi RW Jr, Pipkin T, McCallum $\mathrm{MH}$. Differentiation of cocaine toxicity: role of 
the toxicology drug screen. Drug Intell Clin Pharm 1988; 22: 582-587.

47. Raikos N, Tsouakali H, Psaroulis D, Vassiliadis $\mathrm{N}$, Tsoungas M, Njau SN. Amphetamine derivative related deaths in northerm Greece. Forensic Sci Int 2002; 128: 31-34.

48. Renfroe CL, Messinger TA. Street drug analysis: an eleven year perspective on illicit drug alteration. Semin Adolesc Med 1985; 1: 247 257.

49. Ricaurte GA, Bryan G, Strauss L, Seiden L, Schuster C. Hallucinogic amphetamine selectively destroys brain serotonin nerve terminals. Science 1985; 229: 986-988.

50. Roig Traver A. El éxtasis una revisión del MDMA, MDA y demás feniletilaminas psicoactivas. Premio Socidrogalcohol, 1989.

51. Rothe M, Pragst F, Spiegel K, Harrach T, Fischer K, Kunkel J. Hair concentrations and self-reported abuse history of 20 amphetamine and ecstasy users. Forensic Sci Int 1997; 89: 111-128.

52. Sánchez Senac C, Pérez-Sirvent C, Torres Sánchez C, Luna A. Etude des composants actifs, adultérants et excipients dans des échantillons illicites de drogues de design dans le sud-est de I'Espagne pendant les aneés 1990 et 2001. Comunicación presentada a las XV Jornadas Mediterraneas de Medicina Legal. Terrasini, Sicilia, 2002

53. Sánchez-Ramos JR. Psychostimulants. Neurol Clin 1993; 11: 535-553.

54. Schauben JL. Adulterants and substitutes. Emerg Med Clin North Am 1990; 8: 595-611.
55. Shannon M. Clinical toxicity of cocaine adulterants. Ann Emerg Med 1988; 17: 1243-1247.

56. Smith M. Street drug roulette continues. Emerg Med Serv $2002 ; 31: 30$

57. Tagliaro F, Antonioli C, Moretto S, Archetti S, Ghielmi S, Marigo M. High-sensitivity low-cost methods for determination of cocaine in hair: high-performance liquid chromatography and capillary electrophoresis. Forensic Sci Int 1993; 63: 227-238

58. Traldi P, Favretto D, Tagliaro F. Ion trap mass spectrometry, a new tool in the investigation of drugs of abuse in hair. Forensic Sci Int 1993; 63: 239-252.

59. Vanbisnt R, Koening J, Di Fabio V, Hassoun A. Bile analysis of drugs in post-mortem cases. Forensic Sci Int 2002; 128: 35-40.

60. Windahl KL, McTigue MJ, Pearson JR, Pratt SJ, Rowe JE, Sear EM. Investigation of the impurities found in methamphetamine synthesised from pseudoephedrine by reduction with hydriodic acid and red phosphorus. Forensic Sci Int 1995; 76: 97-114.

61. Winek Ch L, Wahba WW, Winek Jr ChL, Balzer TW. Drug and chemical blood-level data 2001. Forensic Sci Int 2001; 122: 107-123.

62. Wu AH, Forte E, Casella G, Sun K, Hemphill G, Foery $\mathrm{R}$, Schanzenbach $\mathrm{H}$. CEDIA for screening drugs of abuse in urine and the effect of adulterants. J Forensic Sci 1995; 40: 614-618.

63. Yev D, In-Hei H. Toxicity, MDMA. Emedicine, July 12, 2002. 
